\title{
Giant galaxy growing from recycled gas: ALMA maps the circumgalactic molecular medium of the Spiderweb in [C I]
}

\author{
B. H. C. Emonts ${ }^{1}$, M. D. Lehnert ${ }^{2}$, H. Dannerbauer ${ }^{3,4}$, C. De Breuck ${ }^{5}$, \\ M. Villar-Martín ${ }^{6}$, G. K. Miley ${ }^{7}$, J. R. Allison ${ }^{8,9}$, B. Gullberg ${ }^{10}$, \\ N. A. Hatch ${ }^{11}$, P. Guillard ${ }^{2}$, M. Y. Mao ${ }^{12}$, R. P. Norris ${ }^{13,14}$ \\ ${ }^{1}$ National Radio Astronomy Observatory, 520 Edgemont Road, Charlottesville, VA 22903 \\ ${ }^{2}$ Sorbonne Université, CNRS, UMR 7095, Institut d'Astrophysique de Paris, 98bis bvd Arago, 75014, Paris, France \\ ${ }^{3}$ Instituto de Astrofísica de Canarias, E-38205 La Laguna, Tenerife, Spain \\ ${ }^{4}$ Universidad de La Laguna, Dpto. Astrofísica, E-38206 La Laguna, Tenerife, Spain \\ ${ }^{5}$ European Southern Observatory, Karl Schwarzschild Strasse 2, 85748 Garching, Germany \\ ${ }^{6}$ Centro de Astrobiología (INTA-CSIC), Ctra de Torrejón a Ajalvir, km 4, 28850 Torrejón de Ardoz, Madrid, Spain \\ ${ }^{7}$ Leiden Observatory, University of Leiden, P.O. Box 9513, 2300 RA Leiden, Netherlands \\ ${ }^{8}$ Sydney Institute for Astronomy, School of Physics A28, The University of Sydney, NSW 2006, Australia \\ ${ }^{9}$ ARC Centre of Excellence for All-sky Astrophysics in 3 Dimensions (ASTRO 3D) \\ ${ }^{10}$ Centre for Extragalactic Astronomy, Department of Physics, Durham University, Durham DH1 3LE, UK \\ ${ }^{11}$ School of Physics and Astronomy, University of Nottingham, University Park, Nottingham NG7 2RD, UK \\ 12 Jodrell Bank Observatory, University of Manchester, Macclesfield, Cheshire SK11 9DL, UK \\ ${ }^{13}$ CSIRO Astronomy and Space Science, Australia Telescope National Facility, PO Box 76, Epping NSW, 1710, Australia
}

${ }^{14}$ Western Sydney University, Penrith South, NSW 1797, Australia

\begin{abstract}
The circumgalactic medium (CGM) of the massive Spiderweb Galaxy, a conglomerate of merging proto-cluster galaxies at $\mathrm{z}=2.2$, forms an enriched interface where feedback and recycling act on accreted gas. This is shown by observations of $[\mathrm{C} \mathrm{I}], \mathrm{CO}(1-0)$ and $\mathrm{CO}(4-3)$ performed with the Atacama Large Millimeter Array (ALMA) and Australia Telescope Compact Array (ATCA). [C I] and CO(4-3) are detected across $\sim 50 \mathrm{kpc}$, following the distribution of previously detected low-surface-brightness $\mathrm{CO}(1-0)$ across the CGM. This confirms our previous results on the presence of a cold molecular halo. The central radio galaxy MRC 1138-262 shows a very high global $L^{\prime} \mathrm{CO}(4-3) / L^{\prime} \mathrm{CO}(1-0)$ $\sim 1$, suggesting that mechanisms other than FUV-heating by star formation prevail at the heart of the Spiderweb Galaxy. Contrary, the CGM has $L^{\prime}{ }_{\mathrm{CO}(4-3)} / L^{\prime} \mathrm{CO}(1-0)$ and $L^{\prime}{ }_{[\mathrm{CI}]} / L^{\prime} \mathrm{CO}(1-0)$ similar to the ISM of five galaxies in the wider proto-cluster, and its carbon abundance, $X_{[\mathrm{CI}]} / X_{\mathrm{H}_{2}}$, resembles that of the Milky Way and starforming galaxies. The molecular CGM is thus metal-rich and not diffuse, confirming a link between the cold gas and in-situ star formation. Thus, the Spiderweb Galaxy grows not directly through accretion of gas from the cosmic web, but from recycled gas in the GCM.
\end{abstract}

Key words: galaxies: clusters: intracluster medium - galaxies: haloes - galaxies: high-redshift - galaxies: individual: MRC1138-262 - (galaxies:) intergalactic medium

\section{INTRODUCTION}

Most of the baryons in the Universe lie outside galaxies. We can study baryonic halos around galaxies through absorption lines towards distant quasars, or cooling-radiation emitted in Ly $\alpha$. Absorption-line studies detect $~ 100 \mathrm{kpc}$ halos of warm, $\mathrm{T} \sim 10^{4} \mathrm{~K}$, enrich gas around high- $z$ galaxies and quasars (e.g., Prochaska et al. 2014; Neeleman et al. 2017).

We recently discovered that the coldest gas phase can also exist in such environments, by revealing a molecular gas reservoir across the halo of the massive forming Spiderweb
Galaxy at $\mathrm{z}=2.2$ (Emonts et al. 2016, hereafter EM16). The Spiderweb Galaxy is a conglomerate of starforming galaxies that surround the radio galaxy MRC 1138-262, and that are embedded in a giant Ly $\alpha$ halo (Pentericci et al. 1997; Miley et al. 2006). We refer to the entire $200 \mathrm{kpc}$ region of the $\mathrm{Ly} \alpha$ halo as the "Spiderweb Galaxy", because it will likely evolve into a single dominant cluster galaxy (Hatch et al. 2009). The Spiderweb Galaxy is part of a larger proto-cluster (Kurk et al. 2004; Kodama et al. 2007; Dannerbauer et al. 2014).

The halo gas spans a wide range of temperatures 
and densities $\left(\mathrm{T} \sim 100-10^{7} \mathrm{~K}, \mathrm{n} \sim 10^{-3}-10^{4} \mathrm{~cm}^{-3}\right.$; Carilli et al. 2002, EM16). Across the inner $\sim 70 \mathrm{kpc}$, we detected $\sim 10^{11}$ $\mathrm{M}_{\odot}$ of molecular gas via $\mathrm{CO}(1-0)$ (EM16). The location and velocity of the $\mathrm{CO}$, as well as its large angular scale (EM16, their Fig. S1), imply that the bulk of the molecular gas is found in the gaseous medium that lies between the brightest galaxies in the halo. We refer to this gaseous medium as the circumgalactic medium (CGM). There is also diffuse blue light across the halo, indicating that in-situ star formation occurs within the CGM (Hatch et al. 2008). Since the surface densities of the molecular gas and the rate of star formation fall along the Schmidt-Kennicutt relation, the $\mathrm{CO}(1-0)$ results provided the first direct link between star formation and cold molecular gas in the CGM of a forming massive galaxy at high-z (EM16). Extended $\mathrm{CO}$ is also found in the CGM of a massive galaxy at $z=3.47$ (Ginolfi et al. 2017).

Here we present observations sensitive to low-surfacebrightness extended emission of atomic carbon, $\left[\mathrm{C}_{\mathrm{I}}\right]^{3} \mathrm{P}_{1-}{ }^{3} \mathrm{P}_{0}$ (hereafter [C I]) in the CGM of the Spiderweb Galaxy. We supplement these with observations of $\mathrm{CO}(1-0)$ and $\mathrm{CO}(4-$ $3)$ to study the chemical composition and excitation conditions of the gas. $\left[\mathrm{CI}_{\mathrm{I}}\right]$ and $\mathrm{CO}(1-0)$ are fully concomitant in molecular clouds across the Milky Way (Ojha et al. 2001; Ikeda et al. 2002). They have a similar critical density, with the $\left[\mathrm{C}_{\mathrm{I}}\right] J=1$ level well populated down to $\mathrm{T}_{\mathrm{k}} \sim 15 \mathrm{~K}(\mathrm{~Pa}-$ padopoulos et al. 2004). A large positive K-correction means that, at comparable resolution, [C I] is much brighter than $\mathrm{CO}(1-0)$. This becomes progressively more advantageous towards higher redshifts, as the instrumental $\mathrm{T}_{\text {sys }}$ at the corresponding frequencies become more comparable (Papadopoulos et al. 2004; Tomassetti et al. 2014). Furthermore, a high cosmic ray flux from star formation or radio jets may reduce the CO abundance in favor of [C I] (Bisbas et al. 2015, 2017).

We assume $\mathrm{H}_{0}=71 \mathrm{~km} \mathrm{~s}^{-1} \mathrm{Mpc}^{-1}, \Omega_{\mathrm{M}}=0.27$ and $\Omega_{\Lambda}=$ 0.73 , i.e., $8.4 \mathrm{kpc} /{ }^{\prime \prime}$ and $D_{\mathrm{L}}=17309 \mathrm{Mpc}$ at $\mathrm{z}=2.2($ EM16).

\section{OBSERVATIONS}

We observed the Spiderweb for $1.8 \mathrm{hrs}$ on-source during ALMA cycle- 3 on 16 Jan 2016 in its most compact $12 \mathrm{~m}$ configuration (C36-1; baselines $15-161 \mathrm{~m})$. We placed two adjacent spectral windows of $1.875 \mathrm{GHz}$ on $[\mathrm{CI}]{ }^{3} \mathrm{P}_{1-}{ }^{3} \mathrm{P}_{0}$ at $v_{\text {obs }} \sim 155.7 \mathrm{GHz}\left(v_{\text {rest }}=492.16 \mathrm{GHz}\right)$ and another two on $\mathrm{CO}(4-3)$ at $v_{\text {obs }} \sim 145.8 \mathrm{GHz}\left(v_{\text {rest }}=461.04 \mathrm{GHz}\right)$. The ALMA data were reduced in CASA (Common Astronomy Software Applications; McMullin et al. 2007). We binned the data to $30 \mathrm{~km} \mathrm{~s}^{-1}$ channels and cleaned signal $\geq 1 \mathrm{mJy} \mathrm{bm}^{-1}$. We then Hanning smoothed the data to a resolution of 60 $\mathrm{km} \mathrm{s}^{-1}$. The resulting noise is $0.085 \mathrm{mJy}_{\text {beam }}{ }^{-1}$ channel $^{-1}$. We imaged our field using natural weighting out to $\sim 33^{\prime \prime}$, where the primary beam response drops to $\sim 10 \%$ sensitivity. The synthesized beam is $2.3^{\prime \prime} \times 1.5^{\prime \prime}$ with $\mathrm{PA}=73.4^{\circ}$.

Using the ATCA, we made a 2-pointing mosaic with an on-source time of $\sim 90 \mathrm{hrs}$ per pointing at $v_{\mathrm{obs}} \sim 36.5 \mathrm{GHz}$ to observe $\mathrm{CO}(1-0)$. The first pointing was centred on the Spiderweb Galaxy (EM16). The second one was centred $\sim 23^{\prime \prime}$ to the west and observed in Jan 2016 in $750 \mathrm{C}$ configuration for $42 \mathrm{hrs}$ and in April 2016 in H214 configuration for $40 \mathrm{hrs}$ on-source. The observing strategy and data reduction in MIRIAD followed EM16. Because the Spiderweb Galaxy was located near the edge of the primary beam in the second pointing, its strong continuum caused beam-smearing errors that could not be completely eliminated, even with modelbased continuum subtraction in the $(u, v)$-domain (Allison et al. 2012). This prevented us from improving the image of the faint $\mathrm{CO}(1-0)$ in the halo compared with EM16. We imaged both pointings separately using natural weighting, and combined them using the task LINMOS. We binned the channels to $34 \mathrm{~km} \mathrm{~s}^{-1}$ and applied a Hanning smooth, resulting in a resolution of $68 \mathrm{~km} \mathrm{~s}^{-1}$. The noise in the center of the mosaic is $0.073 \mathrm{mJy} \mathrm{bm}^{-1}$, with a beam of $4.7^{\prime \prime} \times 4.1^{\prime \prime}\left(\mathrm{PA} 36.3^{\circ}\right)$. Velocities are in the optical frame relative to $z=2.1612$.

\section{RESULTS}

Fig. 1 shows $[\mathrm{C} \mathrm{I}], \mathrm{CO}(4-3)$ and $\mathrm{CO}(1-0)$ from proto-cluster galaxies within $250 \mathrm{kpc}$ radius around the Spiderweb Galaxy. Six proto-cluster galaxies show line emission (Table 1 ).

The central radio galaxy MRC 1138-262 is covered fully by the ALMA beam and shows an extraordinary high global $L_{\mathrm{CO}(4-3)}^{\prime} / L_{\mathrm{CO}(1-0)}^{\prime} \sim 1$ (Table 1). In metal-rich environments, such high global gas excitation states are hard to achieve with far-UV photons from star formation, and cloud-heating mechanisms due to cosmic rays, jet-induced shocks, or gas turbulence must be prevailing (Papadopoulos et al. 2008, 2012; Ivison et al. 2012). MRC 1138-262 also has a high $L_{[\mathrm{CI}]}^{\prime} / L_{\mathrm{CO}}^{\prime} \sim 0.67$, exceeding that of most submm galaxies (SMGs), quasi-stellar objects (QSOs), and lensed galaxies (Walter et al. 2011; Alaghband-Zadeh et al. 2013; Bothwell et al. 2017). We compare our $\left[\mathrm{C}_{\mathrm{I}}\right]{ }^{3} \mathrm{P}_{1^{-}}{ }^{3} \mathrm{P}_{0}$ detection with $\left[\mathrm{C}_{\mathrm{I}}\right]{ }^{3} \mathrm{P}_{2}-{ }^{3} \mathrm{P}_{1}$ data from Gullberg et al. (2016), which we tapered and smoothed to the same spatial resolution (Fig. 1). We derive a [C I] fine-structure ratio of $L_{[\mathrm{CI}] 2 \rightarrow 1}^{\prime} / L_{[\mathrm{CI}] 1 \rightarrow 0}^{\prime} \sim 0.62$, which implies an excitation temperature $\mathrm{T}_{\mathrm{ex}} \sim 32 \mathrm{~K}$ for optically thin gas (Stutzki et al. 1997).

While the bulk of the $[\mathrm{CI}]$ and $\mathrm{CO}(4-3)$ in the Spiderweb Galaxy is associated with the central radio galaxy MRC 1138-262, we also detect emission across $\sim 50 \mathrm{kpc}$ in the CGM (Fig. 2). As with our previous $\mathrm{CO}(1-0)$ results, the extended $[\mathrm{C} \mathrm{I}]$ is not co-spatial in either location or velocity with ten of the brightest satellite galaxies visible in Fig. 2 (Kuiper et al. 2011; EM16). The [C I] and $\mathrm{CO}(1-0)$ appear to follow the same distribution and kinematics across the velocity range where both lines are reliably detected $(-87$ to $273 \mathrm{~km} \mathrm{~s}^{-1}$ in Fig. 2). At the highest velocities, the $[\mathrm{C} \mathrm{I}]{ }^{3} \mathrm{P}_{1}$ ${ }^{3} \mathrm{P}_{0}$ peaks $\sim 7 \mathrm{kpc}$ SE of the core of the radio galaxy, at a location where previous high-resolution ALMA data found a concentration of [C I ${ }^{3} \mathrm{P}_{2^{-}}{ }^{3} \mathrm{P}_{1}$ (Gullberg et al. 2016). The $\mathrm{CO}(4-3)$ and $[\mathrm{CI}]$ show similar morphologies.

The bright $\left[\mathrm{C}_{\mathrm{I}}\right]$ and $\mathrm{CO}(4-3)$ in the central $\sim 2^{\prime \prime}(\sim 17$ $\mathrm{kpc}$ ) beam make it non-trivial to determine flux densities across the CGM. We therefore taper the ALMA data to a beam of $\sim 8^{\prime \prime}(\sim 70 \mathrm{kpc})$, which covers the full $\mathrm{CO}(1-0)$ halo. We then take the spectrum within this tapered beam and subtract the line profile of the central radio galaxy (Fig. 1). The resulting spectra of the CGM are shown in Fig. 3. For both [C I] and $\mathrm{CO}(4-3), \sim 30 \%$ of the total flux is spread on 17-70 kpc scales (Table 1). The ten bright satellite galaxies with known redshifts, and likely also any fainter satellites (Hatch et al. 2008), do not substantially contribute to this emission. The reasons are that the galaxies have a much higher velocity dispersion than the gas (Fig. 3; Kuiper et al. 

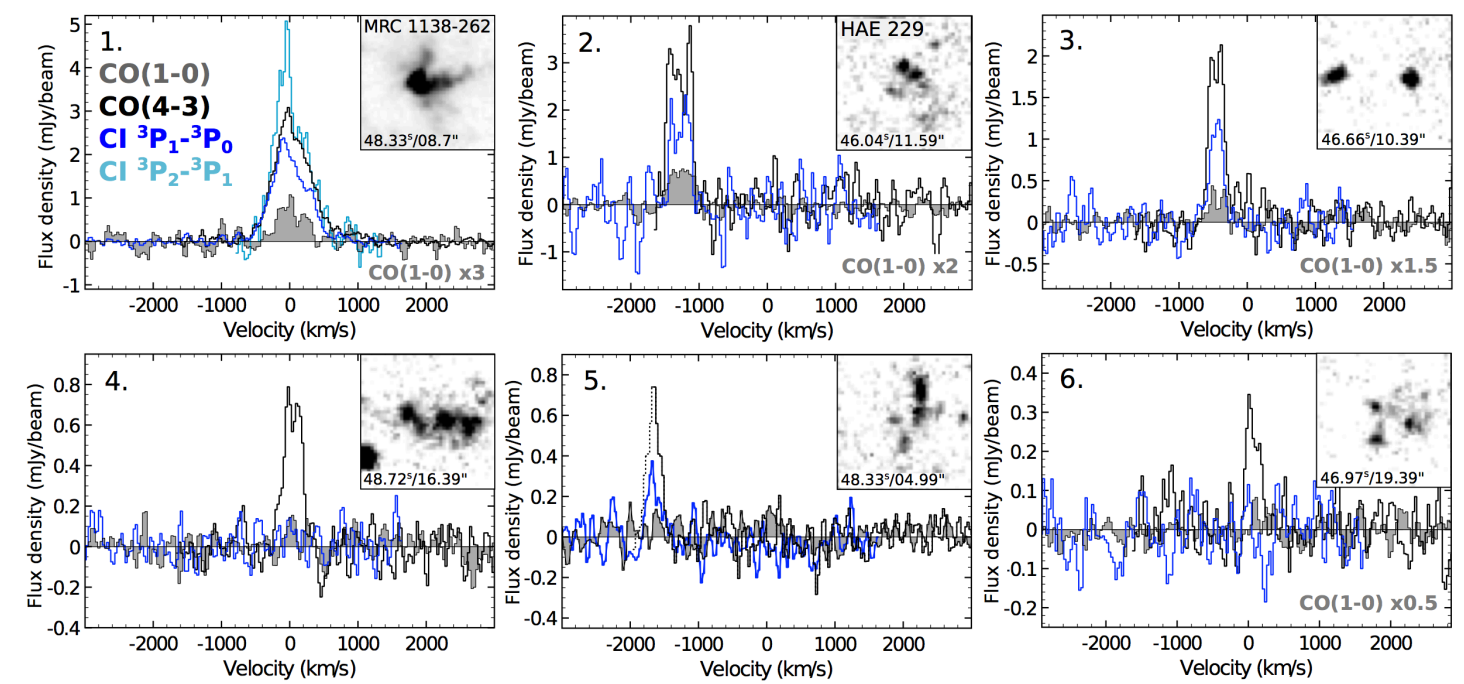

Figure 1. Spectra of $\left[\mathrm{C}_{\mathrm{I}}\right]{ }^{3} \mathrm{P}_{1}{ }^{3} \mathrm{P}_{0}$ (blue), $\mathrm{CO}(4-3)$ (black), and $\mathrm{CO}(1-0)$ (grey) in the six proto-cluster galaxies. Except for radio galaxy MRC 1138-262, all are located far outside the Ly $\alpha$ halo of the Spiderweb Galaxy. Some of the CO(1-0) spectra are scaled up by a factor indicated at the bottom-right, to better visualize them. For MRC 1138-262 we also show the $\left[\mathrm{C}_{\mathrm{I}}\right]{ }^{3} \mathrm{P}_{2}-{ }^{3} \mathrm{P}_{1}$ line (light blue), derived by tapering and smoothing the ALMA data from Gullberg et al. (2016) to the resolution of our $\left[\mathrm{C}_{\mathrm{I}}\right]{ }^{3} \mathrm{P}_{1}{ }^{3}{ }^{3} \mathrm{P}_{0}$ data. Galaxy \#2 is H $\alpha$ emitter HAE 229, for which Dannerbauer et al. (2017) detected CO(1-0) across a large disk. The CO(4-3) line of galaxy \#5 fell at the edge of the band, and the dotted line estimates the profile if it is symmetric. The top-right inset in each panel shows a $2^{\prime \prime} \times 2^{\prime \prime}$ region of the galaxy in $H S T / \mathrm{ACS}$ F $475 \mathrm{~W}+\mathrm{F} 814 \mathrm{~W}$ imaging (Miley et al. 2006). Coordinates in seconds and arcsec are relative to RA=11h40m and $\delta=-26^{\circ} 29^{\prime}$.

Table 1. Emission-line properties. Velocity $v$ is relative to $z=2.1612$, while $v$ and FWHM are derived by fitting a Gaussian function to the $\mathrm{CO}(4-3)$ line ([C I] for galaxy \#5). The ratios of the brightness luminosity $\left(L^{\prime}\right)$ are $\mathrm{r}_{\mathrm{CI} / 1-0}=L_{[\mathrm{CI}] 1 \rightarrow 0}^{\prime} / L_{\mathrm{CO}(1-0)}^{\prime}, \mathrm{r}_{\mathrm{CI} / 4-3}=L_{[\mathrm{CI}] 1 \rightarrow 0}^{\prime} / L_{\mathrm{CO}(4-3)}^{\prime}$, and $\mathrm{r}_{4-3 / 1-0}=L_{\mathrm{CO}(4-3)}^{\prime} / L_{\mathrm{CO}(1-0)}^{\prime}$. The molecular gas mass $\mathrm{M}_{\mathrm{H}_{2}}$ is derived from $L_{\mathrm{CO}(1-0)}^{\prime}$ (Solomon \& Vanden Bout 2005), assuming $\alpha_{\mathrm{CO}}=\mathrm{M}_{\mathrm{H}_{2}} / L_{\mathrm{CO}(1-0)}^{\prime}=0.8 \mathrm{M}_{\odot}\left(\mathrm{K} \mathrm{km} \mathrm{s}^{-1} \mathrm{pc}^{2}\right)^{-1}$ for galaxies \#1-6, and $\alpha_{\mathrm{CO}}=4 \mathrm{M}_{\odot}\left(\mathrm{K} \mathrm{km} \mathrm{s}^{-1} \mathrm{pc}^{2}\right)^{-1}$ for the CGM (see EM16). The [C I] mass $\left(\mathrm{M}_{[\mathrm{CI}]}\right)$ is estimated following Weiß et al. (2005), assuming $T_{\mathrm{ex}}=30 \mathrm{~K}$. Errors (in brackets) include uncertainties in $I$ from both the noise (see Eqn. 2 of Emonts et al. 2014; also Sage 1990) and the absolute flux calibration (5\% for ALMA; 20\% for ATCA).

\begin{tabular}{ccccccccccc}
$\#$ & $\begin{array}{c}v \\
\mathrm{~km} / \mathrm{s}\end{array}$ & $\begin{array}{c}\mathrm{FWHM} \\
\mathrm{km} / \mathrm{s}\end{array}$ & $\boldsymbol{I}_{[\mathrm{CI}] 1 \rightarrow 0}$ & $\begin{array}{c}I_{\mathrm{CO}(1-0)} \\
\mathrm{Jy} / \mathrm{bm} \times \mathrm{km} / \mathrm{s}\end{array}$ & $I_{\mathrm{CO}(4-3)}$ & $\mathrm{r}_{\mathrm{CI} / 1-0}$ & $\mathrm{r}_{\mathrm{CI} / 4-3}$ & $\mathrm{r}_{4-3 / 1-0}$ & $\begin{array}{c}\mathrm{M}_{\mathrm{H}_{2}} \\
10^{10} \mathrm{M}_{\odot}\end{array}$ & $\begin{array}{c}\mathrm{M}_{[\mathrm{CI}]} \\
10^{6} \mathrm{M}_{\odot}\end{array}$ \\
\hline 1 & - & $610(10)$ & $1.32(0.07)$ & $0.11(0.03)^{\dagger}$ & $1.77(0.09)$ & $0.66(0.18)$ & $0.67(0.04)$ & $1.00(0.28)$ & $2.0(0.6)$ & $21(1.0)$ \\
2 & $-1290(10)$ & $375(20)$ & $0.60(0.08)$ & $0.15(0.04)^{\ddagger}$ & $1.19(0.08)$ & $0.22(0.05)$ & $0.44(0.07)$ & $0.49(0.17)$ & $2.8(0.6)$ & $9.5(1.2)$ \\
3 & $-450(10)$ & $255(15)$ & $0.29(0.03)$ & $0.06(0.02)$ & $0.54(0.03)$ & $0.27(0.09)$ & $0.47(0.05)$ & $0.56(0.19)$ & $1.1(0.4)$ & $4.6(0.5)$ \\
4 & $45(10)$ & $310(20)$ & $<0.03$ & $<0.03$ & $0.25(0.02)$ & - & $<0.11$ & $>0.52$ & $<0.6$ & $<0.5$ \\
5 & $-1655(10)$ & $220(15)$ & $0.08(0.01)$ & $<0.03$ & $0.16(0.01)^{*}$ & $>0.15$ & $0.44(0.06)$ & $>0.33$ & $<0.6$ & $1.2(0.1)$ \\
6 & $60(15)$ & $200(25)$ & $<0.04$ & $<0.03$ & $0.13(0.02)$ & - & $<0.27$ & $>0.27$ & $<0.6$ & $<0.6$ \\
$\mathrm{CGM}$ & - & - & $0.56(0.09)$ & $0.11(0.04)$ & $0.79(0.07)$ & $0.28(0.11)$ & $0.62(0.11)$ & $0.45(0.17)$ & $10(4)$ & $8.9(1.4)$ \\
\hline
\end{tabular}

$\dagger$ The ATCA profile in Fig. 1 provides an upper limit to the $\mathrm{CO}(1-0)$ content of MRC 1138-262, because the $\mathrm{CO}(1-0)$ data have a larger beam than the $[\mathrm{CI}]$ and $\mathrm{CO}(4-3)$ data, and therefore include more extended $\mathrm{CO}(1-0)$. The corresponding $I_{\mathrm{CO}(1-0)} \sim 0.126 \mathrm{Jy} \mathrm{bm}^{-1} \times$ $\mathrm{km} \mathrm{s}^{-1}$. To estimate lower limit values, we tapered existing high-resolution VLA data (EM16) to the spatial resolution of our ALMA data. This gives $I_{\mathrm{CO}(1-0)} \gtrsim 0.101 \mathrm{Jy} \mathrm{bm}^{-1} \times \mathrm{km} \mathrm{s}^{-1}$. However, these VLA data have lower sensitivity, hence likely underestimate the full width of the profile. $I_{\mathrm{CO}(1-0)}$ in the Table is a weighted average of the two values, although both values are within the uncertainties. ¥ Dannerbauer et al. (2017) previously reported a somewhat higher $I_{\mathrm{CO}(1-0)}$, although our estimate agrees to within the uncertainties. * $\mathrm{CO}(4-3)$ falls at the edge of the band and half the profile is missing. We derive values assuming that the line profile is symmetric.

2011), and the $3 \sigma$ upper limit for even the brightest satellite galaxies is $I_{[\mathrm{CI}]}<0.028 \mathrm{Jybeam}^{-1} \times \mathrm{km} \mathrm{s}^{-1}(\mathrm{FWHM}=$ $200 \mathrm{~km} \mathrm{~s}^{-1}$ ), or $<5 \%$ of the [C I] brightness of the CGM.

\section{DISCUSSION}

Observations of $[\mathrm{C} \mathrm{I}]{ }^{3} \mathrm{P}_{1-}{ }^{3} \mathrm{P}_{0}, \mathrm{CO}(1-0)$, and $\mathrm{CO}(4-3)$ enable us to estimate the carbon abundance and excitation conditions of the molecular gas in the CGM and proto-cluster galaxies. Fig. 4 (top) shows that the values for $L_{[\mathrm{CI}]}^{\prime} / L_{\mathrm{CO}(4-3)}^{\prime}$ spread across a large range (see also Walter et al. 2011; Alaghband-Zadeh et al. 2013; Bothwell et al. 2017). When instead comparing the ground-transitions of $[\mathrm{C} \mathrm{I}]{ }^{3} \mathrm{P}_{1}-{ }^{3} \mathrm{P}_{0}$ and $\mathrm{CO}(1-0)$, Fig. 4 (bottom) shows two interesting results. First, the CGM has excitation conditions, $L_{\mathrm{CO}(4-3)}^{\prime} / L_{\mathrm{CO}(1-0)}^{\prime}$, and relative $\left[\mathrm{C}_{\mathrm{I}}\right]$ brightness, $L_{[\mathrm{CI}]}^{\prime} / L_{\mathrm{CO}(1-0)}^{\prime}$, similar to those of the proto-cluster galaxies, as well as low- $z$ star-forming galaxies. Second, both the gas excitation and relative [C I] brightness are substantially higher in the radio galaxy MRC 1138-262. A possible explanation for the latter is that 


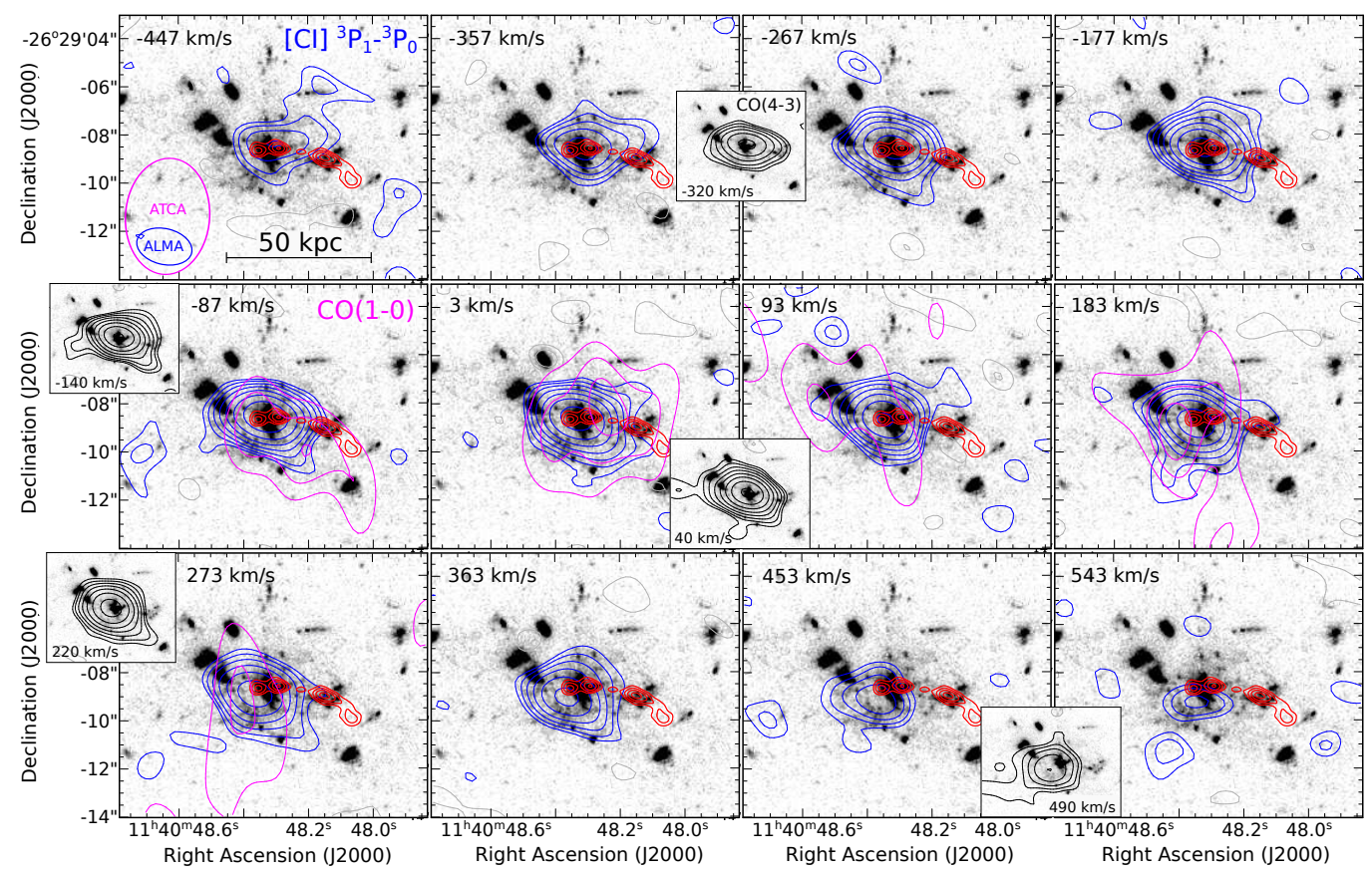

Figure 2. Channels maps of the $[\mathrm{C} I]{ }^{3} \mathrm{P}_{1}-{ }^{3} \mathrm{P}_{0}$ emission (blue contours) over-plotted on an $H S T / \mathrm{ACS}$ F $475 \mathrm{~W}+\mathrm{F} 814 \mathrm{~W}$ image of the Spiderweb Galaxy (Miley et al. 2006). The magenta contours indicate the previously detected $\mathrm{CO}(1-0)$ emission in channels where it is bright enough to be reliably detected (EM16). The most prominent features seen in $\left[\mathrm{C}_{\mathrm{I}}\right]$ and $\mathrm{CO}(1-0)$ are also detected in $\mathrm{CO}(4-3)$ (black contours in the insets). All data-sets were binned to a velocity resolution of $90 \mathrm{~km} \mathrm{~s}^{-1}$, and the central velocity of each channel is indicated. Contour levels of $[\mathrm{CI}]$ and $\mathrm{CO}(4-3)$ start at $2 \sigma$ and increase by factor 1.5 , with $\sigma=0.07 \mathrm{mJy}$ beam ${ }^{-1}$ (negative contours are shown in grey). $\mathrm{CO}(1-0)$ contour levels are at $2,3,4,5 \sigma$, with $\sigma=0.086 \mathrm{mJy}_{\text {beam }}{ }^{-1}$. The red contours indicate the $36 \mathrm{GHz}$ radio continuum (EM16). The synthesized beams of the ALMA and ATCA data are shown in the bottom-left corner of the top-left plot.

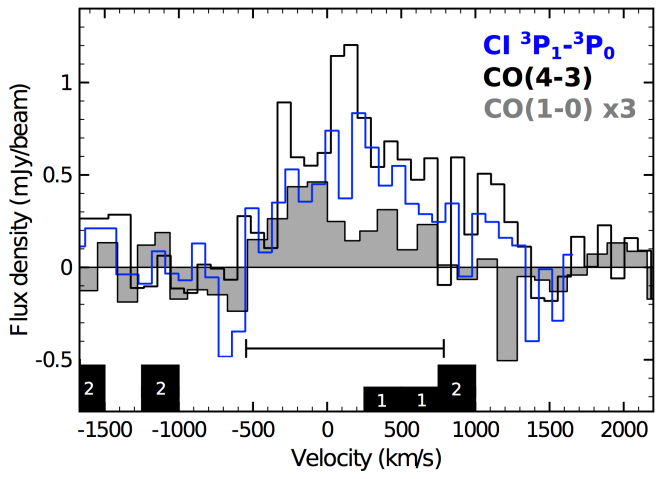

Figure 3. Emission on 17-70 kpc scales in the Spiderweb's CGM. The spectra were extracted by tapering the various data to $\sim 8^{\prime \prime}$ and subtracting the central 2" spectra of MRC 1138-262 (Fig. 1). For the central $\mathrm{CO}(1-0)$ spectrum of MRC 1138-262, we used the average between the untapered ATCA spectrum and a tapered high-resolution VLA spectrum from EM16, as explained in Table 1. The horizontal bar indicates the conservative velocity range over which we detect all three tracers in the CGM, which we used to determine intensities and ratios. The botton histogram shows velocities of satellite galaxies that lie within the molecular halo, based on [O II], [O III] and $\mathrm{H} \alpha$ (Kuiper et al. 2011).

the $\mathrm{CO}(1-0)$ luminosity is reduced due to a high cosmic ray flux near the AGN (Bisbas et al. 2017). Alternatively, the [C I] luminosity may depend on processes that also affect the gas excitation, and thus the luminosity of high- $J$ CO lines like $\mathrm{CO}(4-3)$ (Sect. 3).
We estimate an $\mathrm{H}_{2}$ mass in the CGM on 17-70 kpc scales of $\mathrm{M}_{\mathrm{H}_{2}} \sim 1.0 \pm 0.4 \times 10^{11}\left(\alpha_{\mathrm{CO}} / 4\right) \mathrm{M}_{\odot} \quad$ (Table 1; Solomon \& Vanden Bout 2005). The $\left[\mathrm{C} \mathrm{I}_{\mathrm{I}}\right]$ mass in the $\mathrm{CGM}$ is $\mathrm{M}_{[\mathrm{CI}]} \sim 8.9 \pm 1.4 \times 10^{6} \mathrm{M}_{\odot}$, assuming $\mathrm{T}_{\mathrm{ex}} \sim 30 \mathrm{~K}$ (Weiß et al. 2005). This results in a $[\mathrm{CI}]$ abundance of $X_{[\mathrm{CI}]} / X_{\mathrm{H}_{2}}=\mathrm{M}_{[\mathrm{CI}]} /\left(6 \mathrm{M}_{\mathrm{H}_{2}}\right) \sim 1.5 \pm 0.6 \times 10^{-5}\left(4 / \alpha_{\mathrm{CO}}\right)$, close to that of the Milky Way $\left(\sim 2.2 \times 10^{-5}\right)$ and other high- $z$ starforming galaxies (Frerking et al. 1989; Weiß et al. 2005; Bothwell et al. 2017). The $\mathrm{H}_{2}$ densities must be at least $~ 100$ $\mathrm{cm}^{-3}$, which is the high end of densities of the cool neutral medium, where the $\mathrm{HI}$ to $\mathrm{H}_{2}$ transition occurs (Bialy et al. 2017). More likely, values will be close to $\sim 500 \mathrm{~cm}^{-3}$, the critical density of [C I] (Papadopoulos et al. 2004).

\subsection{Mixing in the CGM}

Our findings of extended [C I], $\mathrm{CO}(1-0)$ and $\mathrm{CO}(4-3)$ imply that the cold molecular CGM is metal-rich and not diffuse. As we showed in EM16, the surface densities of the molecular CGM and the rate of in-situ star formation across the halo fall on the same Kennicutt-Schmidt relation as for starforming galaxies (Kennicutt 1998). The fact that the gas excitation and $[\mathrm{C} \mathrm{I}]$ abundance of the CGM are similar to that of the ISM in star-forming galaxies strengthens this claim.

Despite the similarities between the CGM of the Spiderweb and the ISM in surrounding proto-cluster galaxies, it is unlikely that the cold CGM consists mainly of gas that is currently being tidally stripped from proto-cluster galaxies. If originally the gas was stripped, the low velocity-dispersion of the cold gas compared to that of the galaxies means that 


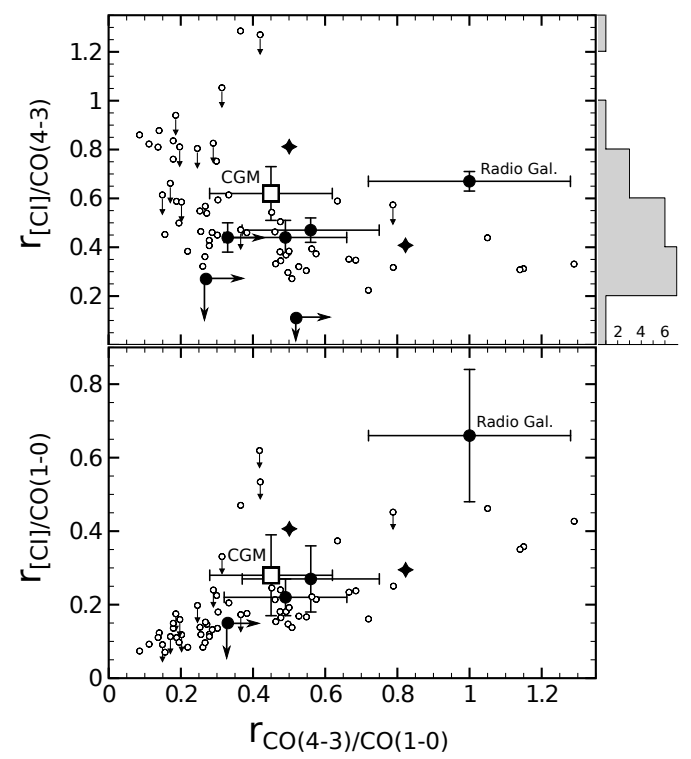

Figure 4. Ratios of the $[\mathrm{C} \mathrm{I}], \mathrm{CO}(1-0)$ and $\mathrm{CO}(4-3)$ lines tracing a wide range of carbon abundances and excitation conditions. The open square represents the CGM of the Spiderweb Galaxy (17-70 $\mathrm{kpc}$ ), the large solid dots the six proto-cluster galaxies from Fig. 1. The two stars represent two high- $z$ lensed SMGs (Danielson et al. 2011; Lestrade et al. 2010, 2011), the small open circles low- $z$. star-forming galaxies (Kamenetzky et al. 2016; Israel et al. 2015; Rosenberg et al. 2015). The histogram on the right ordinate of the top-panel is the $\mathrm{r}_{\mathrm{CI} / \mathrm{CO}(4-3)}$ distribution of high- $z$ SMGs and QSOs (Alaghband-Zadeh et al. 2013; Bothwell et al. 2017).

the gas must have had at least a dynamical time of $\mathrm{t}_{\mathrm{dyn}} \gtrsim 10^{8}$ yr to settle. Since the life-time of the OB stars across the CGM is only $\sim 10^{7} \mathrm{yr}$, they must have formed long after the cold gas settled and cooled (see Hatch et al. 2008).

Our results have important implications for our understanding of galaxy formation. Most importantly, the [C I] and $\mathrm{CO}$ properties do not corroborate models of efficient and direct stream-fed accretion of relatively pristine gas (e.g., Dekel et al. 2009). Instead, they agree with more complex models where the gas in the CGM is a melange from various sources - metal-enriched outflows, mass transfer among galaxies, gas accretion, and mergers (Mori \& Umemura 2006; Narayanan et al. 2015; Anglés-Alcázar et al. 2017; FaucherGiguère et al. 2016). If the gas becomes multiphase and turbulent as it flows, the interaction and mixing of gas from these various sources is likely efficient (Cornuault et al. 2017). The gradual build-up of carbon, oxygen and dust that is starting to be modeled across these extended regions mimics many of the properties that we observe in the CGM of the Spiderweb. Thus, our results support the hypothesis that galaxies grow from recycled gas in the CGM and not directly out of accreted cold gas from the cosmic web.

\section{ACKNOWLEDGMENTS}

We thank Padelis Papadopoulos for valuable feedback and expert advice on how to maximize carbon emissions and pollute the environments of galaxies. This paper makes use of the following ALMA data: ADS/JAO.ALMA\#2015.1.00851.S. ALMA is a partnership of ESO (representing its member states), NSF (USA) and NINS (Japan), together with NRC (Canada), MOST and ASIAA (Taiwan), and KASI (Republic of Korea), in cooperation with the Republic of Chile. The Joint ALMA Observatory is operated by ESO, AUI/NRAO and NAOJ. The Australia Telescope is funded by the Commonwealth of Australia for operation as a National Facility managed by CSIRO. The National Radio Astronomy Observatory is a facility of the National Science Foundation operated under cooperative agreement by Associated Universities, Inc. This research received funding from the Spanish Ministerio de Economía y Competitividad grants Ramón y Cajal RYC2014-15686 (HD) and AYA2015-64346-C2-2-P (MV) and the Australian Research Council Centre of Excellence for All-sky Astrophysics in 3D project CE170100013 (JA).

\section{REFERENCES}

Alaghband-Zadeh S., et al., 2013, MNRAS, 435, 1493

Allison, J. R., et al. 2012, MNRAS, 423, 2601

Anglés-Alcázar D., et al., 2017, MNRAS, 470, 4698

Bialy, S., Burkhart, B., \& Sternberg, A. 2017, ApJ, 843, 92

Bisbas, T. G., Papadopoulos, P. P., \& Viti, S. 2015, ApJ, 803, 37

Bisbas T. G., et al., 2017, ApJ, 839, 90

Bothwell M. S., et al., 2017, MNRAS, 466, 2825

Carilli C. L., et al., 2002, ApJ, 567, 781

Cornuault N., et al., 2017, A\&A in press, arXiv:1609.04405

Danielson, A. L. R., et al. 2011, MNRAS, 410, 1687

Dannerbauer H., et al., 2014, A\&A, 570, A55

Dannerbauer, H., et al. 2017, A\&A, 608, A48

Dekel, A., et al. 2009, Nature, 457, 451

Emonts B. H. C., et al., 2013, MNRAS, 430, 3465

Emonts B. H. C., et al., 2014, MNRAS, 438, 2898

Emonts B. H. C., et al., 2016, Science, 354, 1128

Faucher-Giguère, C.-A., et al. 2016, MNRAS, 461, L32

Frerking, M. A., et al. 1989, ApJ, 344, 311

Ginolfi, M., et al. 2017, MNRAS, 468, 3468

Gullberg, B., et al. 2016, A\&A, 591, 73

Hatch N. A., et al., 2008, MNRAS, 383, 931

Hatch N. A., et al., 2009, MNRAS, 395, 114

Ikeda M., et al., 2002, ApJS, 139, 467

Israel, F., Rosenberg M., van der Werf P. 2015, A\&A, 578, 95

Ivison, R. J., et al. 2012, MNRAS, 425, 1320

Kamenetzky, J., et al. 2016, ApJ, 829, 93

Kennicutt Jr. R. C., 1998, ApJ, 498, 541

Kodama T., et al., 2007, MNRAS, 377, 1717

Kuiper E., et al., 2011, MNRAS, 415, 2245

Kurk J. D., et al., 2004, A\&A, 428, 817

Lestrade, J.-F., et al. 2010, A\&A, 522, L4

Lestrade, J.-F., et al. 2011, ApJL, 739, 30

McMullin, J. P., et al. 2007, ASP Conference Series, 376, 127

Miley G. K., et al., 2006, ApJL, 650, L29

Mori, M., \& Umemura, M. 2006, Nature, 440, 644

Narayanan D., et al., 2015, Nature, 525, 496

Neeleman M., et al., 2017, Science, 355, 1285

Ojha, R., Stark, A. A., Hsieh, H. H., et al. 2001, ApJ, 548, 253

Papadopoulos, et al. 2000, ApJ, 528, 626

Papadopoulos P., Thi W., Viti S., 2004, MNRAS, 351, 147

Papadopoulos, P. P., et al. 2008, A\&A, 491, 483

Papadopoulos, P. P., et al. 2012, MNRAS, 426, 2601

Pentericci L., et al., 1997, A\&A, 326, 580

Prochaska J. X., Lau M., Hennawi J., 2014, ApJ, 796, 140

Rosenberg, M. J. F., et al. 2015, ApJ, 801, 72

Sage L. J., 1990, A\&A, 239, 125

Solomon P. M., Vanden Bout P. A., 2005, ARA\&A, 43, 677

Stutzki, J., et al. 1997, ApJL, 477, L33

Tomassetti, M., et al. 2014, MNRAS, 445, L124

Walter F., et al., 2011, ApJ, 730, 18

Weiß A., Downes D., Henkel C., Walter F., 2005, A\&A, 429, 25 